\title{
Federated Learning Incentive Mechanism Design via Enhanced Shapley Value Method
}

\author{
Xun Yang $\mathbb{D}^{1}$, Weijie Tan $\mathbb{D}^{2,3,4}$ Changgen Peng $\mathbb{D}^{2,3}$ Shuwen Xiang $\mathbb{D}^{1},{ }^{1}$ and Kun Niu $\mathbb{D}^{2}$ \\ ${ }^{1}$ College of Mathematics and Statistics, Guizhou University, Guiyang 550025, China \\ ${ }^{2}$ State Key Laboratory of Public Big Data, College of Computer Science and Technology, Guizhou University, Guiyang 550025, China \\ ${ }^{3}$ Guizhou Big Data Academy, Guizhou University, Guiyang 550025, China \\ ${ }^{4}$ Key Laboratory of Advanced Manufacturing Technology, Ministry of Education, Guizhou University, Guiyang 550025, China
}

Correspondence should be addressed to Weijie Tan; wjtan@gzu.edu.cn and Changgen Peng; cgpeng@gzu.edu.cn

Received 10 July 2021; Revised 20 October 2021; Accepted 9 December 2021; Published 28 January 2022

Academic Editor: James Ying

Copyright (c) 2022 Xun Yang et al. This is an open access article distributed under the Creative Commons Attribution License, which permits unrestricted use, distribution, and reproduction in any medium, provided the original work is properly cited.

Federated learning (FL) is an emerging collaborative machine learning method. In FL processing, the data quality shared by users directly affects the accuracy of the federated learning model, and how to encourage more data owners to share data is crucial. In other words, how to design a good incentive mechanism is the key problem in FL. In this paper, we propose an incentive mechanism based on the enhanced Shapley value method for FL. In the proposed mechanism, the enhanced Shapley value method is proposed to measure income distribution, which takes multiple influence factors as weights. The analytic hierarchy process (AHP) is used to find the corresponding weight value of the influence factors. Finally, the numerical experiments are carried to verify the performance of the proposed incentive mechanism. The results show that compared with the Shapley value method considering the single factor, the income distribution of all participants can better reflect multiple factor contribution when using the enhanced Shapley value method.

\section{Introduction}

With the rapid development of the artificial intelligence technology, it is quite common for cross-organizational and cross-institutional data use. How to ensure data privacy and security while realizing data sharing $[1,2]$ have attracted extensive attention, which is a fundamental challenge in the era of artificial intelligence [3-6]. Federated learning (FL) is a new distributed machine learning method to address the data island problem, and it was first proposed by Google in 2016 [7-9], which can be applied to the Android phone user's local model updating and training between multiple participants or multiple computing nodes. Nowadays, it has been used in all walks of life widely.

In the process of FL, the incentive mechanism is an important research topic [10-12]. To make all data owners contribute their data for model training actively, it is necessary to establish a reasonable incentive mechanism to encourage data owners to share valuable data. A valuable incentive mechanism can enable all participants to continu- ously and carry out efficient model training and improve the final trained federated model more accuracy. When there is no a meaningful incentive mechanism, all participants do not update data in real time in the process of data federation and do not contribute to FL actively, which will not obtain the accurate training model, and all participants dare not use the trained model. It will also cause a waste of time and economic investment of all participants.

Recently, there are a series of research results about the incentive mechanism of FL. In [11], the authors proposed an incentive scheme for a local, client machine learning model for FL, which solves the problem that participants may opt out of the federated system and provides useless information to the federated system without satisfactory incentives. To facilitate collaborative machine learning among multiple model owners, the work in [12] proposed a hierarchical incentive mechanism framework, which solves the problem of hierarchical structure within federated systems. Zhan et al. [13] designed a deep reinforcement learning-based incentive mechanism, which solves unique 
the nonsharing of data and difficult contribution evaluation. $\mathrm{Yu}$ et al. [14] proposed a divide budget incentive method, which solves the restriction of temporary mismatch between contribution and reward in FL. From these researches, we note that participant's submodel influences the quality of trained model generated from FL. Generally, the participants have an enthusiasm to cooperate with each other for improving the quality of model. In $[15,16]$, all participants in FL are considered as having equivalent contributions. However, the contributions of participants have distinct in some cases. For example, the FL model is applied in WeBank, which has cooperated with such financial institutions, insurance companies, and enterprises.

In these existing works, they do not take multiple distribution factors such as cost input, risk factor, and data quality that affect the distribution of benefits. To obtain income distribution fair and just, we proposed an incentive mechanism based on the enhanced Shapley value method for FL. In the proposed method, all federated participants get different federated incomes according the contribution in model training, and there is no free-riding among all participants.

The major contributions of this paper are summarized as the threefold:

(1) We construct the federated incentive model for multiple participants, introduce the cooperative game to the incentive model in FL, and consider multiple factors that affect the income distribution of participants in the FL system

(2) To allocate the income for the federated participants more fair, we propose the analytic hierarchy process (AHP) to construct the expert judgment matrix according the multiple contribution factors, which achieves the corresponding weights of all the influencing factors

(3) The effectiveness of this incentive mechanism is verified through numerical experiments. Experimental results show that the income distribution of all participants can better reflect multiple factor contribution

The rest of this paper is organized as follows: The related work and our main contribution of this paper are introduced in Section 2. The definition of FL, cooperative games, Shapley value, and the AHP are introduced in Section 3. The fair federated model and incentive enhanced Shapley value method are established in Section 4. The rationality of the method is verified by numerical experiments and numerical comparison in Section 5. The conclusion and future work are drawn in Section 6.

\section{Related Work}

The model training accuracy of FL is affected by incentive mechanism, which has been widely concerned at present [15-17]. In [18, 19], the authors proposed an incentive mechanism which combined the contract and reputation can improve the efficiency of federated model training. In [20], an optimization method in the FL wireless networks was formulated, which captures two trade-offs and obtains the global optimal solution by characterizing the closedform solutions to all subproblems. In [21, 22], within the Stackelberg framework, a motivation-based interaction model was established between global servers and participating devices to encourage participants taking part in cooperation. Based on fair allocation for wireless network resources, Li et al. [23] proposed a new objective of resource optimization, which promote a fair society from the perspective of fairness, flexibility, and efficiency. For the linear regression model, the work [24] designed an incentive payment structure to encourage agents provide high-quality data, which can describe the impact of data points on the loss function of the model. The game framework was proposed in $[25,26]$ to solve the efficiency problem of multiparty model.

In the incentive mechanism, the return fairness is a worthy studying problem. The work of [27] studied the dynamics of coalition formation under the bounded rationality condition, which considers the situation that the profit of each team is given by the submodular function, and proposed a profit distribution scheme based on the concept of marginal utility. Xiong et al. [28] proposed an incentive mechanism for the multiattribute user selection, which effectively improves sensing user quality for mobile crowdsensing. The Shapley value method is used to distribute fair the benefits generated in the process of the coalition [29], which is the most widely used method to evaluate fairness $[30,31]$. In [32], the authors combined the quality estimation with a monetary incentive and used the Shapley value method to determine the cost of each household, which was based on the effect of goodness in saving and remaining points. In [33], a repertoire of efficient algorithms was proposed to meet the time-consuming challenge in the Shapley value requiring process. Liu et al. [34] proposed a blockchain-based FedCoin peer-to-peer payment incentive mechanism for FL and used the Shapley value method to distribute federated revenue.

In the existing works, they do not take multiple related factors such as cost input, risk factor, and data quality that affect the distribution of benefits. In order to fully consider the impact of multiple factors on the fairness of distribution, an incentive mechanism for the FL system is proposed to achieve fairness and justice, which uses the enhanced Shapley value method. The proposed incentive mechanism introduces the cooperative game to the incentive model and considers multiple factors that affect the income distribution of participants. The analytic hierarchy process (AHP) is used to construct the expert judgment matrix, which achieves the corresponding weights of all the influencing factors.

\section{Preliminaries}

3.1. Definition of Federated Learning. Let $\{1,2, \cdots, N\}$ be defined as $N$ data owners, and they all hope to merge their respective data $\left\{D_{1}, D_{2}, \cdots, D_{N}\right\}$ to train a machine learning model. A model $M_{\mathrm{SUM}}$ is trained by a traditional method, which puts all data $D=D_{1} \cup D_{2} \cup \cdots \cup D_{N}$ together. A model $M_{\mathrm{FED}}$ can be cooperatively trained by the data owners of 
claimed FL system in learning process, which the data $D_{i}$ never leaked to other participant via any data owner $i$ in this process. Moreover, the precision of $M_{\mathrm{FED}}$, defined as $V_{\mathrm{FED}}$, should be pretty close to the property of $M_{\mathrm{SUM}}$ and $V_{\mathrm{SUM}}$. Generally, if there is a $\delta \geq 0$ satisfies the condition

$$
\left|V_{\mathrm{FED}}-V_{\mathrm{SUM}}\right|<\delta
$$

we say that the FL algorithm has $\delta$-accuracy loss [4].

3.2. Cooperative Games. The $G(N, v)$ is defined cooperative game and satisfies two conditions as follows $[29,35,36]$ :

$$
\begin{gathered}
v\left(S_{1}\right)+v\left(S_{2}\right) \leq v\left(S_{1} \cup S_{2}\right), \\
S_{1} \cap S_{2}=\varnothing, v(\varnothing)=0,
\end{gathered}
$$

where $N$ is a finite set of participants, $S_{1}, S_{2} \in 2^{N}, v: 2^{N}$ $\longrightarrow R$ is characteristic function, $2^{N}$ is the set of all the subsets of $N$. Let $v(S)$ is participants' income function, $v(N)$ indicates the coalition income and $\varphi_{i}(v)$ (defined in formula (6)) is the income of participant $i$ in $v(N)$, which satisfy two constraints:

$$
\begin{aligned}
& v(N)=\sum_{i=1}^{n} \varphi_{i}(v), \\
& v(S) \leq \sum_{i \in S} \varphi_{i}(v),
\end{aligned}
$$

Formula (4) is named as collective rationality, which indicates that the sum of all participants' incomes from the maximum income in $G(N, v)$ should be equivalent to $v(N)$ , and formula (5) is claimed coalition rationality, which shows that the sum of profit allocated to all probable coalitions must be greater than or equivalent to $v(S)$. When $|S|=1$, the formula (5) is individual rationality.

3.3. Shapley Value. Shapley value was presented by Shapley $[29,37]$ in the cooperative game theory, which can effectively solve the problem of cooperative income distribution and define as the following equation:

$$
\begin{gathered}
\varphi_{i}(v)=\sum_{i \in S, S \subset N} w(|S|)[v(S)-v(S \backslash i)], \\
w(|S|)=\frac{(n-|S|) !(|S|-1) !}{n !}
\end{gathered}
$$

where $S \subset N, i=1,2, \cdots, n,|S|$ is the quantity of participants in subset $S, w(|S|)$ is weight coefficient, $v(S)$ is the income of subset $S$ and satisfies Equations (2) and (3), the term $v(S)-v(S \backslash i)$ evaluates the marginal contribution of $i$ to the coalition $S$, and $v(S \backslash i)$ shows the benefit of other players in subset $S$ other than $i$.

\section{Federated Learning Incentive Mechanism}

Before establishing the FL incentive model, we make the following assumptions:
(1) All participants have the ability to pay for FL, and in the process of income distribution, they adopt the best income distribution scheme.

(2) All participants are willing to participate in the coalition and do not withdraw from each sub-coalition, and all parties are very satisfied with the final income distribution scheme.

(3) All participants are completely trustworthy, and there is no cheating.

(4) FL should adopt multiparty agreement to recognize the income distribution scheme to ensure the smooth implementation of the strategy.

4.1. FL Incentive Model. The proposed FL incentive model is as shown in Figure 1, and in this model, we assume that there are $n$ factors and $n$ participants and establish their federated models. We use different colors to represent the process of different factors for different participants, the direction of model updating, and income distribution in the whole FL process.

Step 1. Under the influence of factor 1 , factor $2, \ldots$, and factor $n$ on the federated income, we distribute model 1 , model $2, \ldots$, and model $n$ to participant 1 , participant $2, \ldots$, and participant $n$ and then train the $n$ submodels respective of data participants. The data of the $n$ participants do not leave the local, to protect the privacy of the data in their respective databases.

Step 2. Every time the model runs, the parameters of the $n$ submodels are updated, until the end of the model run, and the models are aggregating to get the federated aggregation model we need.

Step 3. Every time the model runs, using formula (19), each participant $i$ will receive the income $\tilde{\varphi}_{i}(v, t)$ from the federated expected to cost $b(t)$ in round $t$.

Step 4. When the model is running finished, the parameters of all submodels are aggregating to get the final aggregated federated model. Finally, the federated income is allocated according to the enhanced Shapley value method, and each participant $i$ will achieve income $\varphi_{i}^{\prime}(v, t)$ from formula (20).

The income distribution of each participant $i$ is affected by $n$ factors, which are allocated to rely on the contribution of each participant $i$ to the whole federation. This design makes participants get the distributed federated benefits more fairly and get an accurate federated model. Because the influencing factors impact income of participants, the enthusiasm of participants will be affected and, finally, impact the accuracy of the federated model. If each participant $i$ actively cooperates in the federated process and tries to take part in the FL model to update data in the local database in real time, the parameters of the model training will also be updated, and the final federated aggregation model will also be updated. This cycle will continue until the end of the whole model training. Finally, the total federated 


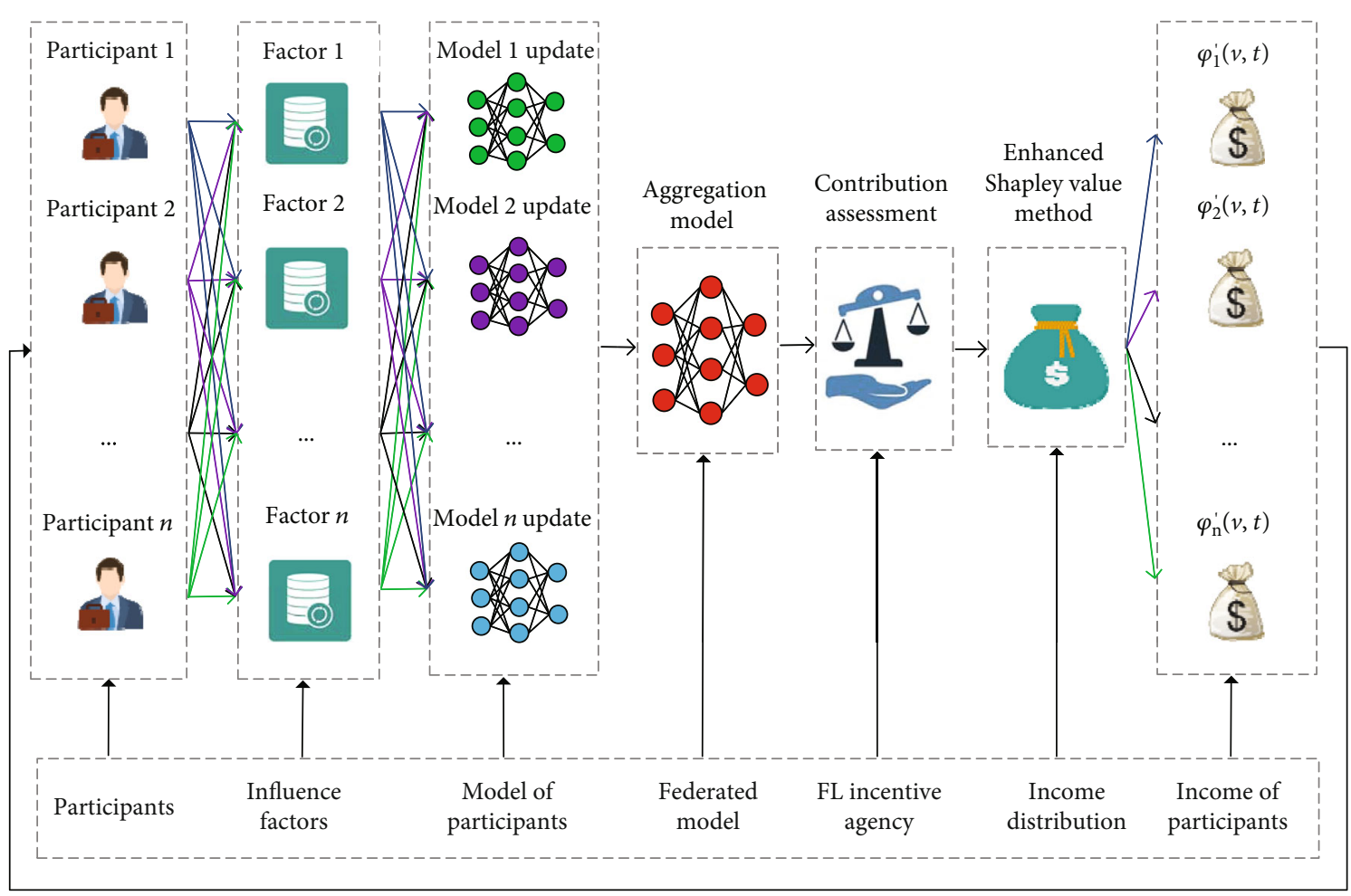

Figure 1: Federated learning incentive model.

income generated is distributed according to the enhanced Shapley value method.

4.2. Contribution Assessment. To encourage the participants to actively participate in the FL, we set each participant to be treated fairly based on their contribution and meet three fair conditions at the same time:

(1) Contribution Fairness. Each participant's income is related to its contribution to the federation.

(2) Expectation Fairness of Risk-Taking. The expected risk and time risk between each participant are minimized.

(3) Expectation Fairness. The expected and time risks taken from each participant vary as little as possible.

We denote $c_{i}(t)$ the cost of participant $i$ and $q_{i}(t)$ the contribution, and $q_{i}(t) \geq 0$ includes risk-taking, data quality, effort degree, and cost input. And we shall use the procurement auction method [41] to estimate $c_{i}(t)$. Further, we define the expected risk $f_{i}(t)$ and time risk $g_{i}(t)$ by the following dynamic system:

$$
\begin{aligned}
& f_{i}(t+1)=\max \left[f_{i}(t)+c_{i}(t)-\varphi_{i}^{\prime}(v, t), 0\right], \\
& g_{i}(t+1)=\max \left[g_{i}(t)+\mu_{i}(t)-\varphi_{i}^{\prime}(v, t), 0\right],
\end{aligned}
$$

where $\mu_{i}(t)$ represents an indicator function is

$$
\mu_{i}(t)= \begin{cases}\tilde{c}_{i}(t), & f_{i}(t)>0 \\ 0, & f_{i}(t) \leq 0 .\end{cases}
$$

Here, when $f_{i}(t)>0$, the time series $g_{i}(t)$ will increase, and $\tilde{c}_{i}(t)$ shows that participant $i$ is the average cost of contribution for the federation. So we have a federally maximized $H$.

$$
H=\frac{1}{T} \sum_{t=1}^{T} \sum_{i=1}^{N}\left[q_{i}(t) \varphi_{i}^{\prime}(v, t)\right]
$$

satisfying fair condition (1), where $T$ is the total quantity of runs of the model and $q_{i}(t)$ represents the contribution made by participant $i$ to the federation. We should treat all participants fairly to minimize their expected loss and distribution. Therefore, we introduce the Lyapunov function formulated as follows [42]:

$$
L(t)=\frac{1}{2} \sum_{i=1}^{N}\left[f_{i}^{2}(t)+g_{i}^{2}(t)\right]
$$

For the convenience of calculation, the root operator is omitted calculation, which will not change the good properties of formula (11). With the change of time, the expected risk deviation of the participants is as follows: 


$$
\begin{aligned}
\Delta L(t)= & \frac{1}{T} \sum_{t=1}^{T}[L(t+1)-L(t)] \\
= & \frac{1}{T} \sum_{t=1}^{T} \sum_{i=1}^{N}\left[\frac{1}{2} f_{i}^{2}(t+1)-\frac{1}{2} f_{i}^{2}(t)+\frac{1}{2} g_{i}^{2}(t+1)-\frac{1}{2} g_{i}^{2}(t)\right] \\
\leq & \frac{1}{T} \sum_{t=1}^{T} \sum_{i=1}^{N}\left[f_{i}(t) c_{i}(t)-f_{i}(t) \varphi_{i}^{\prime}(v, t)+\frac{1}{2} c_{i}^{2}(t)\right. \\
& -c_{i}(t) \varphi_{i}^{\prime}(v, t)+\frac{1}{2} \varphi_{i}^{\prime 2}(v, t)+g_{i}(t) \mu_{i}(t) \\
& \left.-g_{i}(t) \varphi_{i}^{\prime}(v, t)+\frac{1}{2} \mu_{i}^{2}(t)-\mu_{i}(t) \varphi_{i}^{\prime}(v, t)+\frac{1}{2} \varphi_{i}^{\prime 2}(v, t)\right] .
\end{aligned}
$$

Since $\varphi_{i}^{\prime}(v, t)$ is a control variable of the income function of the participant $i$, we extract the formula containing it from formula (12) and get the following result:

$$
\Delta L(t) \leq \frac{1}{T} \sum_{t=1}^{T} \sum_{i=1}^{N}\left\{\dot{\varphi}_{i}^{2}(v, t)-\varphi_{i}^{\prime}(v, t)\left[f_{i}(t)+c_{i}(t)+g_{i}(t)+\mu_{i}(t)\right]\right\} .
$$

The expected loss $\Delta L(t)$ is the distribution function of $f_{i}(t)$ and $g_{i}(t)$. If $\Delta L(t)$ is minimized and fair conditions (2) and (3) are satisfied, the expected loss will gradually decrease with time. Let

$$
F(t)=\varepsilon H-\Delta L(t)
$$

is the target number, which indicates the inequality of expected loss and waiting time between participants, and it should be minimized, where $\varepsilon$ is a federated regularization term to constraint the equilibrium between the two targets. Therefore, the federation is aimed at the following function:

$$
F(t)=\frac{1}{T} \sum_{t=1}^{T} \sum_{i=1}^{N}\left\{\varphi_{i}^{\prime}(v, t)\left[\varepsilon q_{i}(t)+f_{i}(t)+c_{i}(t)+g_{i}(t)+\mu_{i}(t)\right]-\iota^{\prime} \varphi_{i}^{2}(v, t)\right\},
$$

and the constraint condition is

$$
\sum_{i=1}^{N} \tilde{\varphi}_{i}(v, t) \leq b(t), \tilde{\varphi}_{i}(v, t) \geq 0, \text { for } \forall i, t
$$

where $b(t)$ means the cost of participants in the current payment budget, which is determined by their expected loss and the time they spend waiting for the reward to be fully paid, and the $\tilde{\varphi}_{i}(t)$ represents the income that participant $i$ received from the federation in round $t$. Let us take the derivative of formula (15) with respect to $\varphi_{i}^{\prime}(v, t)$, and set the first derivative equal to 0 to obtain $\varphi_{i}^{\prime}(v, t)$ :

$$
\varphi_{i}^{\prime}(v, t)=\frac{1}{2}\left[\varepsilon q_{i}(t)+f_{i}(t)+c_{i}(t)+g_{i}(t)+\mu_{i}(t)\right] .
$$

Moreover, by using the second derivatives of (15) with respect to $\varphi_{i}^{\prime}(t)$, we obtain the following result:

$$
\frac{d^{2}}{\varphi_{i}^{2}(v, t)} F(t)=-1<0
$$

When the federated model runs in the round $t$, the contribution of the participants to the federation is $q_{i}(t)$, and the total compensation of the participants $i$ is $\varphi_{i}^{\prime}(v, t)=$ $1 / 2\left[\varepsilon q_{i}(t)+f_{i}(t)+c_{i}(t)+g_{i}(t)+\mu_{i}(t)\right]$. If budget $b(t)$ is not enough to fully pay all participants' compensation in round $i$, the federated organization will pay them in installments within a certain period. The federated organization will pay $\tilde{\varphi}_{i}(v, t)$ in installments in round $i$ according to the following equation:

$$
\tilde{\varphi}_{i}(v, t)=\frac{\varphi_{i}^{\prime}(v, t)}{\sum_{i=1}^{N} \varphi_{i}^{\prime}(v, t)} b(t)
$$

For the income distribution scheme in Figure 1, when $f_{i}(t)$ and $g_{i}(t)$ are equal to 0 , this is a very important condition, and participant $i$ does not invest extra cost; then, $\varphi_{i}^{\prime}(v, t)=\varepsilon q_{i}(t)$. After that, participant $i$ will use the enhanced Shapley value method to evaluate its contribution based on the federation and allocate the future income. This income allocation method will first consider the participants whose expected risk is not equal to 0 and also consider the contributions of other participants to the federation. According to Figure 1 and the above theoretical analysis, we present the pseudo-code of FL incentive in Algorithm 1.

4.3. The Enhanced Shapley Value Method. To ensure that all participants are satisfied with the federated income distribution scheme and make the distribution mechanism play an incentive role, all participants can actively contribute to the federated model. The Shapley value method considers that the contribution degree of each participant as $1 / n$ ignores the influence of other factors. Therefore, it is necessary to consider the effects of multiple factors on federal income distribution, by taking multiple influence factors as weights, and the enhanced Shapley value method is proposed to measure income distribution $[28,40]$.

$$
\begin{gathered}
\varphi_{i}^{\prime}(v, t)=\varphi_{i}(v, t)+v(N) \times \Delta P_{i}, \Delta P_{i}=P_{i}-\frac{1}{n}, i=1,2, \cdots, n, \\
P_{i}=\left(\alpha_{i 1}, \alpha_{i 2}, \cdots, \alpha_{\text {in }}\right)\left(w_{1}, w_{2}, \cdots, w_{n}\right)^{T}, i=1,2, \cdots, n,
\end{gathered}
$$

where $\varphi_{i}^{\prime}(v, t)$ shows the anticipated income for federated model $i$ after improvement and under a federated game condition in the $t$ round, $v(N)$ is the revenue of the grand coalition, $\varphi_{i}(v, t)$ is the income distribution of participant $i$ using the Shapley value method in the $t$ round of FL. In particular, when $t=1, \varphi_{i}^{\prime}(v, t)$ is calculated using formula 6 , and 


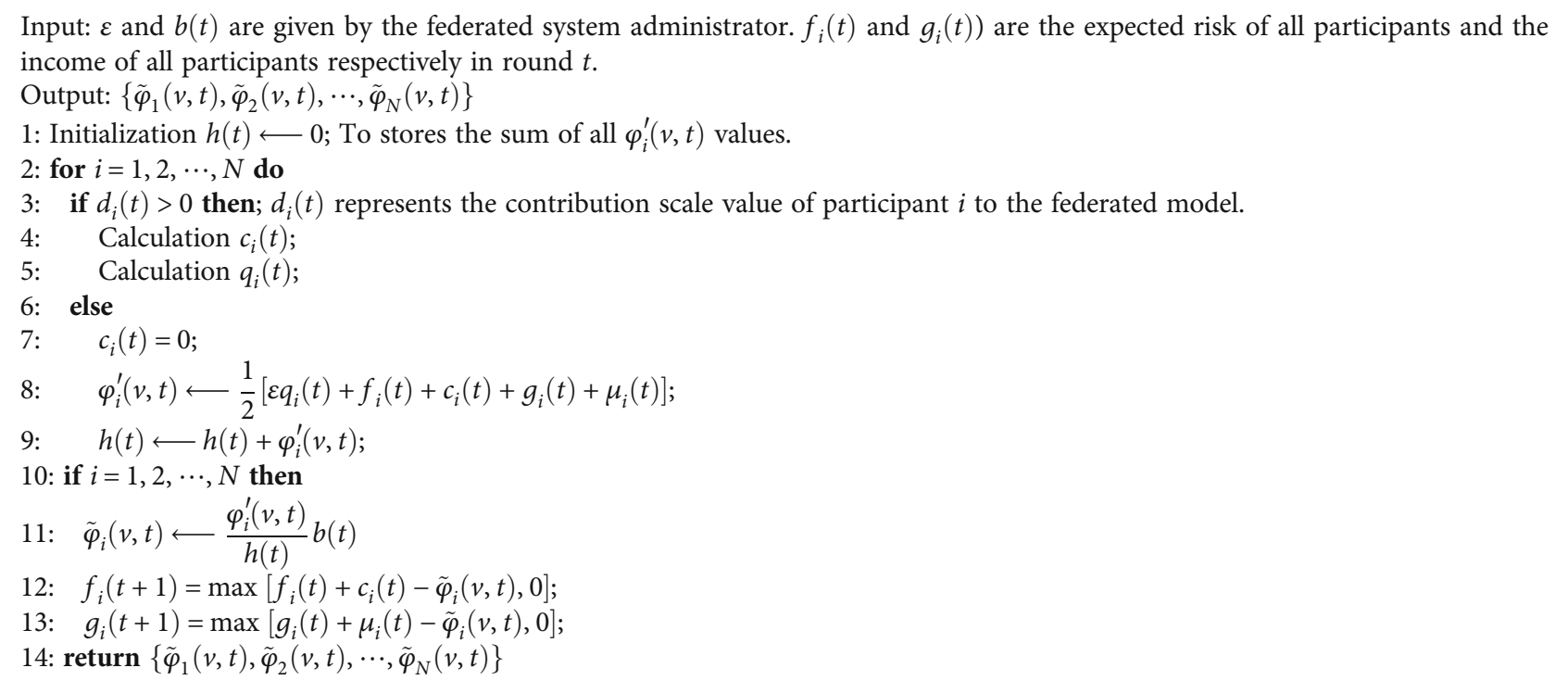

Algorithm 1: FL incentive mechanism via enhanced Shapley value method.

$\Delta P_{i}$ indicates the difference between the comprehensive evaluation factor $P_{i}$ and the average factor $1 / n$ introduced by participant $i$, and it refers to the comprehensive factor as an incentive factor in the federated distribution of incomes.

$$
\Delta P_{i}= \begin{cases}\text { Participant } i \text { should be encouraged, } & P_{i}-\frac{1}{n}>0, \\ \text { Participant } i \text { should be punished, } & P_{i}-\frac{1}{n}<0 .\end{cases}
$$

$P_{i}$ is the sum of the product of the measured values $\alpha_{i 1}$, $\alpha_{i 2}, \ldots, \alpha_{\text {in }}$ of the $n$ factors and the corresponding factor weight $w_{1}, w_{2}, \ldots, w_{n}$, and $\sum_{i=1}^{n} P_{i}=1$. Thus, we have

$$
\left\{\begin{array}{l}
\sum_{i=1}^{n} \Delta P_{i}=0 \\
\sum_{i \in S} \Delta P_{i} \geq \frac{v(S)-\sum_{i \in S} \varphi_{i}(v, t)}{v(S)}
\end{array}\right.
$$

We solve these weights of $n$ influence factors $\alpha_{i 1}, \alpha_{i 2}, \ldots$, $\alpha_{\text {in }}$ with the AHP. It is easy to verify that formula (20) satisfies formulas (4) and (5).

Proof. According to formulas (4) and (23), formula (20) can be proofed as follows:

$$
\begin{aligned}
\sum_{i=1}^{n} \varphi_{i}^{\prime}(v, t) & =\sum_{i=1}^{n}\left[\varphi_{i}(v, t)+v(N) \times \Delta P_{i}\right] \\
& =\sum_{i=1}^{n} \varphi_{i}(v, t)+v(N) \sum_{i=1}^{n} \Delta P_{i} \\
& =v(N) .
\end{aligned}
$$

Hence, formula (20) satisfies formula (4). In addition, according to formulas (5) and (23), formula (20) is proofed as the following:

$$
\begin{aligned}
\sum_{i \in S} \varphi_{i}^{\prime}(v, t) & =\sum_{i \in S}\left[\varphi_{i}(v, t)+v(S) \times \Delta P_{i}\right] \\
& =\sum_{i \in S} \varphi_{i}(v, t)+v(S) \sum_{i \in S} \Delta P_{i} \\
& \geq v(S) .
\end{aligned}
$$

Hence, formula (20) satisfies formula (5).

To solve the Shapley value correction factor of the federated income distribution, the AHP is used to assign the importance degree according to the scale of 1-9. The explanation of scale 1-9 is presented in Table 1 [39].

In Table 1, index indicates the intensity of importance on an absolute scale. The judgment matrix in this paper is constructed by the joint score of the expert group, and they score according to the rules in Table 1. These experts have a strong background in economics, computer, and mathematics. Hence, according to the joint score of the expert group, the judgment matrix $A\left(a_{i j}\right)_{n \times n}$ is obtained.

$$
A=\left[\begin{array}{cccc}
a_{11} & a_{12} & \cdots & a_{1 n} \\
a_{21} & a_{22} & \cdots & a_{2 n} \\
\vdots & \vdots & \vdots & \vdots \\
a_{n 1} & a_{n 2} & \cdots & a_{n n}
\end{array}\right],
$$

and satisfied $a_{i j}>0, a_{j i}=1 / a_{i j}$, and $a_{i i}=1$. The matrix $B$ $\left(b_{i j}\right)_{n \times n}$ is given by normalizing, and according to each column of the matrix $A\left(a_{i j}\right)_{n \times n}, b_{i j}=a_{i j} / \sum_{i, j=1}^{n} a_{i j}, \alpha_{i 1}, \alpha_{i 2}, \cdots$, $\alpha_{\text {in }}$, comes from each $i$-th row element of matrix $B\left(b_{i j}\right)_{n \times n}$ 
TABLE 1: The explanation of index in expert judgment matrix.

\begin{tabular}{lc}
\hline Index & Explanation \\
\hline 1 & The factors $i$ and $j$ are equally essential \\
2 & The factor $i$ is more essential than $j$ slightly \\
3 & The factor $i$ is more essential than $j$ obviously \\
4 & The factor $i$ is more essential than $j$ strongly \\
5 & The factor $i$ is more essential than $j$ extremely \\
$2,4,6,8$ & The index between adjacent indexes above \\
Reciprocal & The value $a_{i j}$ is obtained by factor $i$ and factor $j$, \\
& and the value $a_{j i}=1 / a_{i j}$ is obtained by $j$ \\
& comparing factor with factor $i$
\end{tabular}

and represents the measurement value of $n$ factors affecting federated revenue; $\alpha_{i j}$ represents the $j$-th factor affecting the individual's federated income, and $\sum_{i, j=1}^{n} \alpha_{i j}=1$.

$$
B=\left[\begin{array}{cccc}
b_{11} & b_{12} & \cdots & b_{1 n} \\
b_{21} & b_{22} & \cdots & b_{2 n} \\
\vdots & \vdots & \vdots & \vdots \\
b_{n 1} & b_{n 2} & \cdots & b_{n n}
\end{array}\right] .
$$

Sum matrix $B\left(b_{i j}\right)_{n \times n}$ is according to row vector, $c_{i}=\sum_{i, j=1}^{n} b_{i j}$, and achieves the vector $C$.

$$
C=\left(c_{1}, c_{2}, \cdots, c_{n}\right),
$$

and then, normalizing the vector $C, \quad w_{i}=c_{i} / \sum_{i=1}^{n} c_{i}$. Consequently, the weight value $w$ of judgment matrix $A\left(a_{i j}\right)_{n \times n}$ is obtained.

$$
w=\left(w_{1}, w_{2}, \cdots, w_{n}\right)^{T}, \sum_{i=1}^{n} w_{i}=1
$$

Since the judgment matrix $A\left(a_{i j}\right)_{n \times n}$ is established based on the experience and estimation of experts, some differences will appear between the judgment matrix $A$ and the real situation, and it needs to test the consistency of the matrix $A$ $\left(a_{i j}\right)_{n \times n}$. If the matrix $A\left(a_{i j}\right)_{n \times n}$ is consistent, its eigenvector $w=\left(w_{1}, w_{2}, \cdots, w_{n}\right)^{T}$ can truly reflect the reasons that affect federated income. In this case, we can use the random consistency index (RI) $[38,39]$ in Table 2.

$$
\left\{\begin{array}{l}
\lambda_{\max }=\sum_{i=1}^{n} \frac{\left(A w_{i}\right)}{n w_{i}}, \\
\mathrm{CI}=\frac{\left(\lambda_{\max }-n\right)}{(n-1)}, \\
\mathrm{CR}=\frac{\mathrm{CI}}{\mathrm{RI}},
\end{array}\right.
$$

where $\lambda_{\max }$ is the largest eigenvalue of the matrix $A\left(a_{i j}\right)_{n \times n}$, the $\mathrm{CI}$ represents public consistency index, RI is the average
TABLE 2: Random consistency index.

\begin{tabular}{lccccccccc}
\hline Order & 1 & 2 & 3 & 4 & 5 & 6 & 7 & 8 & 9 \\
\hline RI & 0.00 & 0.00 & 0.52 & 0.89 & 1.11 & 1.25 & 1.35 & 1.4 & 1.45 \\
\hline
\end{tabular}

random consistency index, and CR is the consistency ratio. As we refer to Table 2, matrix $A\left(a_{i j}\right)_{n \times n}$ needs to be satisfied with the consistency.

$\mathrm{CR}= \begin{cases}\text { The matrix } A\left(a_{i j}\right)_{n \times n} \text { is just what we need, } & \frac{\mathrm{CI}}{\mathrm{RI}}<0.1, \\ \text { The matrix } A\left(a_{i j}\right)_{n \times n} \text { needs to be adjusted, } & \frac{\mathrm{CI}}{\mathrm{RI}}>0.1 .\end{cases}$

\section{Numerical Results}

We assume that there are three banks: bank 1, bank 2, and bank 3, which are taking part in the FL model. The incomes of three banks not participating in FL are $v(1)=150$, $v(2)=250$, and $v(3)=350$. If bank 1 and bank 2 coalition can make income $v(1,2)=400$, bank 1 and bank 3 coalition can make income $v(1,3)=800$, bank 2 and bank 3 coalition can make income $v(2,3)=700$, and banks 1,2 , and 3 coalition can make income $v(1,2,3)=1500$. After the three banks unite together, the income distribution of each bank is solved.

5.1. Illustrating Result of the Shapley Value Method. In this subsection, the Shapley value method does not consider any factors to the federated income but distribute the income according to the average distribution method. Therefore, we can calculate the income distribution value of three banks after FL according to the relevant knowledge of cooperative game theory and Equations (6) and (7). The details are shown in Table 3.

Therefore, add the last row of Table 3, and we achieve the federated income value allocated by bank 1 which is the following: $\varphi_{1}(v, t)=416.67$; similarly, the federated income value allocated by bank 2 is as follows: $\varphi_{2}(v, t)=$ 416.67 , and the federated income value allocated by bank 3 is as follows: $\varphi_{3}(v, t)=666.67$.

5.2. Illustrating Result of the Enhanced Shapley Value Method. In the FL incentive model, the income distribution of the federated bank depends not only on the marginal contribution but also on other factors that affect the total income. In this example, we mainly consider the risk factor, data quality, effort degree, and cost input. For banks with higher risk, higher input data quality, higher level of cooperation, and perhaps higher-cost investment, the weight of benefit distribution should be appropriately increased. According to the enhanced Shapley value model and the following AHP flow chart in Figure 2, experts give scores to the influence factors according to Table 1 , and we achieve the judgment matrix $A, B_{1}, B_{2}, B_{3}, B_{4}$. Here, matrix $A$ is the weight matrix of reference layer $B$ to target layer.

Through the weight matrix $A$, the weight value of reference layer $B$ to target layer $A$ can be calculated. Similarly, we 
TABLE 3: Bank 1 federated income calculation.

\begin{tabular}{lcccc}
\hline$S$ & $\{1\}$ & $\{1,2\}$ & $\{1,3\}$ & $\{1,2,3\}$ \\
\hline$v(S)$ & 150 & 400 & 800 & 1500 \\
$v(S \backslash\{1\})$ & 0 & 250 & 350 & 700 \\
$v(S)-v(S \backslash\{1\})$ & 150 & 150 & 450 & 800 \\
$|S|$ & 1 & 2 & 2 & 3 \\
$w(|S|)$ & $1 / 3$ & $1 / 6$ & $1 / 6$ & $1 / 3$ \\
$w(|S|)[v(S)-v(S \backslash\{1\})]$ & $150 / 3$ & $150 / 6$ & $450 / 6$ & $800 / 3$ \\
\hline
\end{tabular}

can obtain the weight matrix $B_{1}, B_{2}, B_{3}, B_{4}$ of scheme layer $C$ to reference layer $B$ and the corresponding weight. These weight values of influence factors and the corresponding $\lambda_{\text {max }}$, and CR values are solved in the following sheet.

Suppose three banks construct judgment matrix according to their own actual situation, and applying to formulas (20)-(31), we achieve the following conclusions:

(1) Expert judgment matrix $A-B$ and weight $w$

\begin{tabular}{ccccc}
\hline$A$ & $B_{1}$ & $B_{2}$ & $B_{3}$ & $B_{3}$ \\
\hline$B_{1}$ & 1 & 1 & $1 / 3$ & $1 / 5$ \\
$B_{2}$ & 1 & 1 & $1 / 4$ & $1 / 3$ \\
$B_{3}$ & 3 & 4 & 1 & $1 / 4$ \\
$B_{4}$ & 5 & 3 & 4 & 1
\end{tabular}$\quad w=\left(\begin{array}{l}0.0918 \\
0.1040 \\
0.2546 \\
0.5496\end{array}\right)$

$, \lambda_{\max }=4.2556, C I=0.0852, C R=0.0957<0.1 ;$

(2) Expert judgment matrix $B_{1}-C$ and weight $w_{1}^{(1)}$

\begin{tabular}{cccc}
\hline$B_{1}$ & $C_{1}$ & $C_{2}$ & $C_{3}$ \\
\cline { 1 - 3 }$C_{1}$ & 1 & 5 & 3 \\
$C_{2}$ & $1 / 5$ & 1 & $1 / 4$ \\
$C_{3}$ & $1 / 3$ & 4 & 1 \\
\hline
\end{tabular}

(3) Expert judgment matrix $B_{2}-C$ and weight $w_{2}^{(1)}$

\begin{tabular}{cccc}
\hline$B_{2}$ & $C_{1}$ & $C_{2}$ & $C_{3}$ \\
\cline { 1 - 3 }$C_{1}$ & 1 & 7 & 7 \\
$C_{2}$ & $1 / 7$ & 1 & 2 \\
$C_{3}$ & $1 / 7$ & $1 / 2$ & 1 \\
\hline
\end{tabular},$w_{2}(1)=\left(\begin{array}{c}0.7732 \\
0.1392 \\
0.0877\end{array}\right), \lambda_{\max }=3.0536, C I=0.0268, C R=0.0516<0.1 ;$

(4) Expert judgment matrix $B_{3}-C$ and weight $w_{3}{ }^{(1)}$

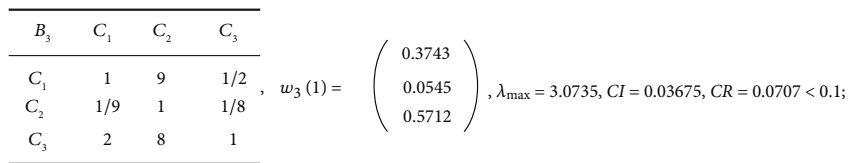

(5) Expert judgment matrix $B_{4}-C$ and weight $w_{4}{ }^{(1)}$

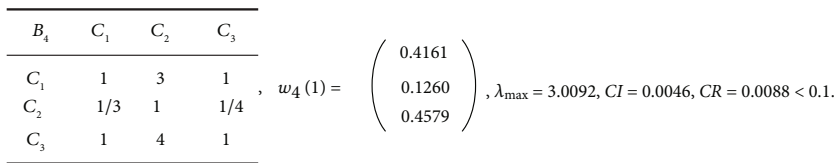

Consequently, we let

$w^{(1)}=\left(w_{1}^{(1)}, w_{2}^{(1)}, w_{3}^{(1)}, w_{4}^{(1)}\right)=\left[\begin{array}{cccc}\alpha_{11} & \alpha_{12} & \alpha_{13} & \alpha_{14} \\ \alpha_{21} & \alpha_{22} & \alpha_{23} & \alpha_{24} \\ \alpha_{31} & \alpha_{32} & \alpha_{33} & \alpha_{34}\end{array}\right]$,

and then, using formula (30), we can calculate

$$
\begin{aligned}
P & =w^{(1)} w \\
& =\left[\begin{array}{llll}
0.6267 & 0.7732 & 0.3747 & 0.4161 \\
0.0936 & 0.1392 & 0.0545 & 0.1260 \\
0.2797 & 0.0877 & 0.5712 & 0.4579
\end{array}\right]\left[\begin{array}{l}
0.0918 \\
0.1040 \\
0.2546 \\
0.5496
\end{array}\right] \\
& =\left[\begin{array}{l}
0.4620 \\
0.1062 \\
0.4319
\end{array}\right],
\end{aligned}
$$

and $\quad \Delta P_{i}=P_{i}-1 / n=(0.4620,0.1062,0.4319)^{T}-1 / 3=$ $(0.1287,-0.2271,0.0986)^{T}$. According to formula (22), $\Delta P_{2}<0$ means that the second bank's enthusiasm in the FL process is significantly lower than the average level of the federated banks and should be punished. Therefore, using formulas (20) and (21) can calculate the income distribution of the three banks in FL as follows:

$$
\begin{aligned}
& \varphi_{1}^{\prime}(v, t)=\frac{2500}{6}+1500 \times 0.1287=609.72, \\
& \varphi_{2}^{\prime}(v, t)=\frac{2500}{6}+1500 \times(-0.2271)=76.02, \\
& \varphi_{3}^{\prime}(v, t)=\frac{4000}{6}+1500 \times 0.0986=814.57 .
\end{aligned}
$$

Through the numerical calculation of the enhanced Shapley method, we achieve the expected result which is the following:

$$
\sum_{i=1}^{3} \varphi_{i}^{\prime}(v, t)=609.72+76.02+814.57 \approx 1500 .
$$

After verification, it is not difficult to find that in the case of the income distribution scheme of the enhanced Shapley value method, the income of the three banks is consistent with the theory.

5.3. Numerical Comparison. In this subsection, we compare the results of income distribution using the Shapley value 


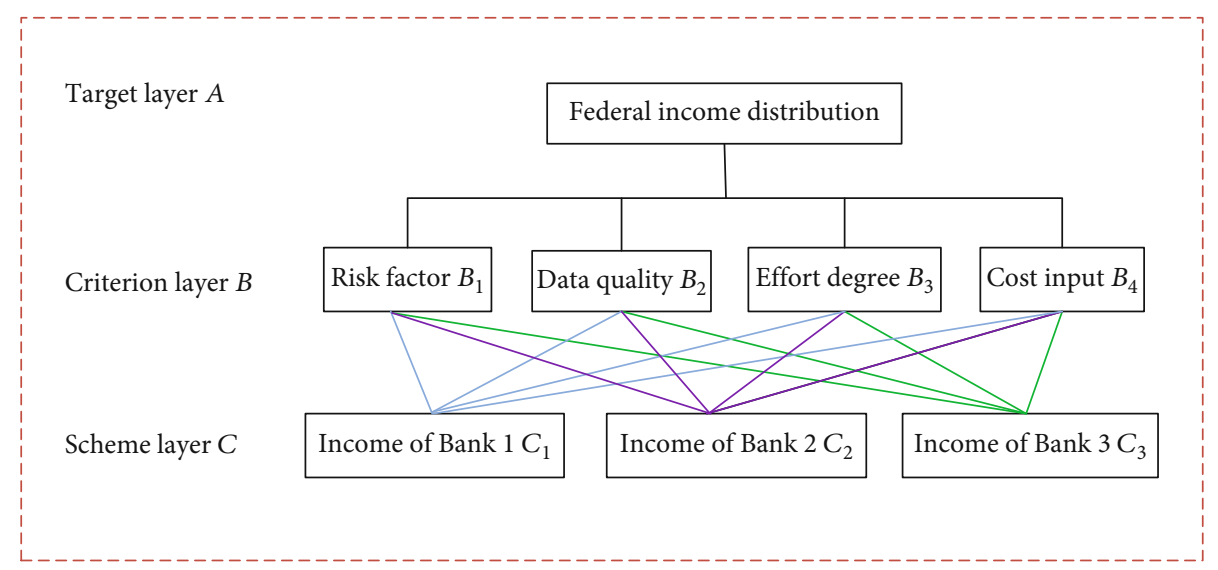

Figure 2: AHP flow chart.

TABLE 4: Income comparison.

\begin{tabular}{lcccc}
\hline Participant & \multicolumn{2}{c}{$\begin{array}{c}\text { Shapley value method } \\
\text { Income distribution }\end{array}$} & \multicolumn{2}{c}{$\begin{array}{c}\text { Enhanced Shapley value method } \\
\text { Enhanced weight }\end{array}$} \\
\hline Bank 1 & 0.3333 & 416.67 & 0.4620 & 609.72 \\
Bank 2 & 0.3333 & 416.67 & 0.1062 & 76.02 \\
Bank 3 & 0.3333 & 666.67 & 0.4319 & 814.57 \\
\hline
\end{tabular}

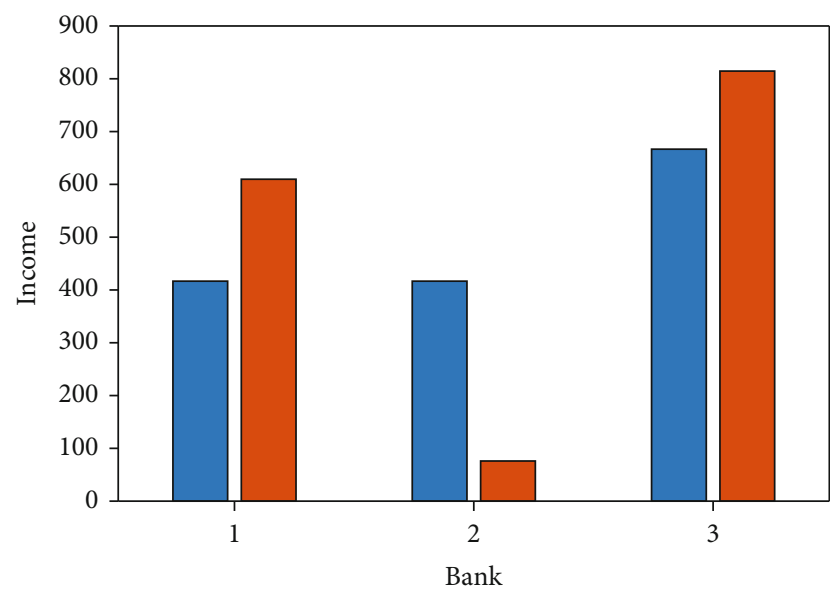

Shapley Value method

Enhanced Shapley Value method

Figure 3: Income histogram of federated model.

method with the results of income distribution using the enhanced Shapley value method [40] in Table 4 and simulate their values in Figure 3.

Table 4 indicates that the Shapley value method does not consider any factors and the corresponding weights in the income distribution; however, in the enhanced Shapley value method, we consider the factors that affect income distribution and the corresponding weights. It is not difficult to find out bank 1 has the largest weight, indicating that bank 1 is the most positive in the whole FL process, followed by the third bank, and the second bank is the worst, which should be punished.

From Figure 3, we can clearly see that income allocated by the Shapley value method shows that the first bank and the second bank have the same income. However, relying on the enhanced Shapley value method, it is easy to find that the second bank is the least profitable.

\section{Conclusion and Future Work}

Federated learning (FL) is a distributed machine learning framework, and how to design an incentive mechanism for federated participants to sustain participate in model training is very important to train an accurate federated model. In this paper, we propose an incentive mechanism using the enhanced Shapley value method, and this incentive mechanism considers four factors that affect the federated income distribution, which can better reflect the fairness of income distribution. Further, the analytic hierarchy process is used to calculate the corresponding weight of four factors. Numerical results verify that the income distribution of all participants can better reflect multiple factor contribution when using the incentive mechanism with the enhanced Shapley value method, which gets a better incentive for federated participants.

In the next step, we will apply the proposed incentive mechanism to solve the problem of participants' income distribution in FL train models. This incentive mechanism can be applied in WeBank, such as financial institutions, insurance companies, and enterprises, for reducing loan risks and improving economic earning significantly in practice. 


\section{Data Availability}

The data used to support the finding of this study are included in the article.

\section{Conflicts of Interest}

The authors declare that there is no conflict of interest regarding the publication of this paper.

\section{Acknowledgments}

This work is supported by the National Natural Science Foundation of China (No. U1836205), the National Natural Science Foundation of China (No. 71961003), the Science and Technology Program of Guizhou Province (No. Guizhou-ScienceContract-Platform-Talent [2020]5017), the Natural Science Foundation of Department of Education of Guizhou Province (Nos. Qian-Education-Contact-Telent [2013]09 and QianEducation-Contact-KY [2021]140), the 13th Five-Year National Cryptography Development Foundation (No. MMJJ20170129), the Research Project of Guizhou University for Talent Introduction (No. [2020]61), the Cultivation Project of Guizhou University (No. [2019]56), and the Open Fund of Key Laboratory of Advanced Manufacturing Technology, Ministry of Education, GZUAMT2021KF[01].

\section{References}

[1] J. B. Xiong, R. Ma, L. Chen et al., "A personalized privacy protection framework for mobile crowdsensing in IIoT," IEEE Transactions on Industrial Informatics, vol. 16, no. 6, pp. 4231-4241, 2020.

[2] Q. Li, B. Xia, H. P. Huang, Y. H. Zhang, and T. Zhang, "TRAC: traceable and revocable access control scheme for mHealth in 5G-enabled IIoT," IEEE Transactions on Industrial Informatics, vol. 14, no. 8, pp. 1551-3203, 2021.

[3] K. Bonawitz, H. Eichner, W. Grieskamp et al., "Towards federated learning at scale: system design," 2019, http://arxiv.org/ abs/1902.01046.

[4] Q. Yang, Y. Liu, T. J. Chen, and Y. X. Tong, "Federated machine learning," ACM Transactions on Intelligent Systems and Technology, vol. 10, no. 2, pp. 1-19, 2019.

[5] T. Li, A. K. Sahu, A. Talwalkar, and V. Smith, "Federated learning: challenges, methods, and future directions," IEEE Signal Processing Magazine, vol. 37, no. 3, pp. 50-60, 2020.

[6] T. Li, A. K. Sahu, M. Zaheer, M. Sanjabi, A. Talwalkar, and V. Smith, "Federated optimization in heterogeneous networks," Conference on Machine Learning and Systems, vol. 2, pp. 429-450, 2020.

[7] J. Konecn, H. B. McMahan, D. Ramage, and P. Richt, "Federated optimization: distributed machine learning for ondevice intelligence," 2016, http://arxiv.org/abs/1610.02527.

[8] J. Konečný, M. M. HB, F. X. Yu, P. Richtárik, A. T. Suresh, and D. Bacon, "Federated learning: strategies for improving communication efficiency," 2016, http://arxiv.org/abs/1610.05492.

[9] H. B. McMahan, E. Moore, D. Ramage, and B. A. Y. Arcas, "Federated learning of deep networks using model averaging," 2016, http://arxiv.org/abs/1602.05629.

[10] J. B. Xiong, X. H. Chen, Q. Yang, L. Chen, and Z. Q. Yao, “A task-oriented user selection incentive mechanism in edge- aided mobile crowdsensing," IEEE Transactions on Network Science and Engineering, vol. 7, no. 4, pp. 2347-2360, 2020.

[11] Y. Liu and J. H. Wei, "Incentives for federated learning: a hypothesis elicitation approach," 2020, http://arxiv.org/abs/ 2007.10596.

[12] W. Y. B. Lim, Z. H. Xiong, C. Miao et al., "Hierarchical incentive mechanism design for federated machine learning in mobile networks," IEEE Internet of Things Journal, vol. 7, no. 10, pp. 9575-9588, 2020.

[13] Y. F. Zhan, P. Li, Z. Qu, D. Zeng, and S. Guo, "A learningbased incentive mechanism for federated learning," IEEE Internet of Things Journal, vol. 7, no. 7, pp. 6360-6368, 2020.

[14] H. Yu, Z. L. Liu, Y. Liu et al., "A sustainable incentive scheme for federated learning," IEEE Intelligent Systems, vol. 35, no. 4, pp. 58-69, 2020.

[15] P. Kairouz, M. M. HB, B. Avent et al., "Advances and open problems in federated learning," 2019, http://arxiv.org/ abs1912.04977.

[16] H. Yu, Z. Liu, Y. Liu et al., "A fairness-aware incentive scheme for federated learning," in Proceedings of the AAAI/ACM Conference on AI, Ethics, and Society, pp. 393-399, New York, NY, USA, 2020.

[17] J. Xiong, R. Ma, L. Chen, Y. Tian, L. Lin, and B. Jin, “Achieving incentive, security, and scalable privacy protection in mobile crowdsensing services," Wireless communications and mobile computing, vol. 2018, Article ID 8959635, 12 pages, 2018.

[18] J. Kang, Z. H. Xiong, D. Niyato, S. Xie, and J. S. Zhang, "Incentive mechanism for reliable federated learning: a joint optimization approach to combining reputation and contract theory," IEEE Internet of Things Journal, vol. 6, no. 6, pp. 10700-10714, 2019.

[19] J. Kang, Z. H. Xiong, D. Niyato, H. Yu, Y. C. Liang, and D. Kim, "Incentive design for efficient federated learning in mobile networks: a contract theory approach," in 2019 IEEE VTS Asia Pacific Wireless Communications Symposium (APWCS), Singapore, 2019.

[20] N. H. Tran, W. Bao, A. Y. Zomaya, M. N. H. Nguyen, and C. Hong, "Federated learning over wireless networks: optimization model design and analysis," in IEEE INFOCOM 2019IEEE Conference on Computer Communications, Paris, France, 2019.

[21] L. U. Khan, S. R. Pandey, N. H. Tran et al., "Federated learning for edge networks: resource optimization and incentive mechanism," IEEE Communications Magazine, vol. 58, no. 10, pp. 88-93, 2020.

[22] Y. Sarikaya and O. Ercetin, "Motivating workers in federated learning: a Stackelberg game perspective," IEEE Networking Letters, vol. 2, no. 1, pp. 23-27, 2020.

[23] T. Li, M. Sanjabi, and V. Smith, "Fair resource allocation in federated learning," 2020, http://arxiv.org/abs1905.10497.

[24] A. Richardson, A. F. Ratsikas, and B. Faltings, "Rewarding high-quality data via influence functions," 2019, http://arxiv .org/abs/1908.11598.

[25] Z. Chen, Z. Liu, K. L. Ng, H. Yu, Y. Liu, and Q. Yang, A Gamified Research Tool for Incentive Mechanism Design in Federated Learning, Springer, Cham, 2020.

[26] J. B. Xiong, M. F. Zhao, M. Z. A. Bhuiyan, L. Chen, and Y. L. Tian, "An ai-enabled three-party game framework for guaranteed data privacy in mobile edge crowdsensing of IoT," IEEE Transactions on Industrial Informatics, vol. 17, no. 2, pp. 922-933, 2021. 
[27] J. E. Augustine, N. Chen, E. Elkind, A. Fanelli, N. Gravin, and D. Shiryaev, "Dynamics of profit-sharing games," Internet Mathematics, vol. 11, no. 1, pp. 1-22, 2015.

[28] J. Xiong, X. Chen, Y. Tian, R. Ma, L. Chen, and Z. Yao, "MAIM: a novel incentive mechanism based on multiattribute user selection in mobile crowdsensing," IEEE Access, vol. 6, pp. 65384-65396, 2018.

[29] L. S. Shapley, “17. A Value for n-Person Games," Contributions to the Theory of Games, vol. 2, no. 28, pp. 307-317, 1953.

[30] T. S. Song, Y. X. Tong, and S. Y. Wei, "Profit allocation for federated learning," in 2019 IEEE International Conference on Big Data (Big Data), Los Angeles, CA, USA, 2019.

[31] G. Wang, "Interpret federated learning with Shapley values," 2019, http://arxiv.org/abs/1905.04519.

[32] S. Yang, F. Wu, S. J. Tang, X. Gao, B. Yang, and G. Chen, "On designing data quality-aware truth estimation and surplus sharing method for mobile crowdsensing," IEEE Journal on Selected Areas in Communications, vol. 35, no. 4, pp. 832847, 2017.

[33] R. Jia, D. Dao, B. Wang et al., "Towards efficient data valuation based on the Shapley value," 2019, http://arxiv.org/abs/ 1902.10275.

[34] Y. Liu, Z. Ai, S. Sun, S. Zhang, Z. Liu, and H. Yu, Fedcoin: A Peer-to-Peer Payment System for Federated Learning, Springer, Cham, 2020.

[35] R. Aumann and B. Peleg, "Von Neumann-Morgenstern solutions to cooperative games without side payments," Bulletin of the American Mathematical Society, vol. 66, no. 3, pp. 173-179, 1960.

[36] R. J. Aumann and J. H. Dreze, "Cooperative games with coalition structures," International Journal of Game Theory, vol. 3, no. 4, pp. 217-237, 1974.

[37] D. Butnariu, "Stability and Shapley value for an n-persons fuzzy game," Fuzzy Sets and Systems, vol. 4, no. 1, pp. 63-72, 1980.

[38] R. W. Saaty, "The analytic hierarchy process-what it is and how it is used," Mathematical Modelling, vol. 9, no. 3-5, pp. 161-176, 1987.

[39] T. Saaty, "How to make a decision: the analytic hierarchy process," European Journal of Operational Research, vol. 48, no. 1, pp. 9-26, 1990.

[40] E. Kalai and D. Samet, "On weighted Shapley values," International Journal of Game Theory, vol. 16, no. 3, pp. 205-222, 1987.

[41] D. Mishra and D. Veeramani, "Vickrey-Dutch procurement auction for multiple items," European Journal of Operational Research, vol. 180, no. 2, pp. 617-629, 2007.

[42] M. J. Neely, "Stochastic network optimization with application to communication and queueing systems," Synthesis Lectures on Communication Networks, vol. 3, no. 1, 2010. 